\title{
MINIMUM VOLUME DESIGN OF FRAMEWORKS AND DISCS FOR ALTERNATIVE LOADING SYSTEMS*
}

\author{
By H. S. Y. CHAN (Oxford University)
}

The problem of minimum volume design of frameworks to carry alternative loading systems was first studied in [1], but the condition for optimum was found only later in [2]. By following the terminology of [3] for designing with elastic-plastic materials, this condition can be stated as follows. If a structure $S$ can be found which is just at the point of collapse under loading system $F_{i}^{1}$ in a deformation mode $u_{i}^{1}$ and which is also at the point of collapse under the loading system $F_{i}^{2}$ in a deformation mode $u_{i}^{2}$ such that the virtual work $D$ per unit volume satisfies

$$
\begin{aligned}
D\left(\epsilon_{i i}^{1}\right)+D\left(\epsilon_{i i}^{2}\right) & =\text { ef }=\text { constant in } S, \\
& \leq \text { ef elsewhere, }
\end{aligned}
$$

then the design $S$ is an optimum, where $\epsilon_{i j}^{1}, \epsilon_{i j}^{2}$ are the virtual strains corresponding to the deformations $u_{i}^{1}, u_{i}^{2}$, and the positive constants $e, f$ can be regarded as reference strain and stress respectively.

It is intended to show that, in the case of frameworks, the above condition can be obtained by the duality theorem of linear programming. An example of optimum design in plane stresses will also be given below.

1. Frameworks with yield stresses $f_{t}$ and $f_{c}$ in tension and compression designed to carry either loads $F_{i}^{1}$ or $F_{i}^{2}$ can be obtained by the method of linear programming [4]. If the cross section areas and lengths of all possible members of the framework are denoted by $a_{i}$ and $l_{i}$ and the end loads in the members are $T_{i}^{1}$ and $T_{i}^{2}$ under the load systems, the problem is formulated as

Minimize

$$
V=\sum_{i} i_{i} a_{i}
$$

subject to

$$
\begin{aligned}
-f_{c} a_{i} \leq & T_{i}^{1} \leq f_{i} a_{i}, \quad a_{i} \geq 0 \\
-f_{c} a_{i} \leq & T_{i}^{2} \leq f_{t} a_{i}, \\
& \sum_{i} b_{i} T_{i}^{1}=F_{i}^{1} \\
& \sum_{i} b_{i j} T_{i}^{2}=F_{i}^{2} ;
\end{aligned}
$$

where (4) are the equations of equilibrium at each joint of the structure, $b_{i ;}$ are therefore direction cosines of the axes of the possible members. The formulation (2)-(4) is a standard linear programming problem, the corresponding dual problem is found to be (see [5]):

*Received November 7, 1966; revised manuscript received January 9, 1967. 
Maximize

$$
W=\frac{1}{e f} \sum_{i}\left(u_{i}^{1} F_{i}^{1}+u_{i}^{2} F_{i}^{2}\right),
$$

subject to

$$
\begin{gathered}
\sum_{i} b_{i j} u_{i}^{1}=\delta_{1 j}^{+}-\delta_{1 i}^{-}, \\
\sum_{i} b_{i j} u_{i}^{2}=\delta_{2 i}^{+}-\delta_{2 i}^{-}, \\
f_{l}\left(\delta_{1 i}^{+}+\delta_{2 i}^{+}\right)+f_{c}\left(\delta_{1 i}^{-}+\delta_{2 i}^{-}\right) \leq e f l_{j}, \quad \delta_{1 i}^{+}, \delta_{1 i}^{-}, \delta_{2 i}^{+}, \delta_{2 i}^{-} \geq 0 ;
\end{gathered}
$$

where $e, f$ are positive constants and the variables are now $u_{i}^{1}, u_{i}^{2}, \delta_{1 j}^{+}, \delta_{1 i}^{-}, \delta_{2 i}^{+}, \delta_{2 j}^{-}$.

The duality theorem of linear programming [5] tells that if either problem (2)-(4) or problem (5)-(7) has solution, then both problems have solution and the following relations will be satisfied by the optimum solutions:

$$
\begin{gathered}
\sum_{i} a_{i} l_{i}=V_{\min }=W_{\max }=\frac{1}{e f} \sum_{i}\left(u_{i}^{1} F_{i}^{1}+u_{i}^{2} F_{i}^{2}\right), \\
\left(f_{t} a_{i}-T_{i}^{1}\right) \delta_{1 i}^{+}=0, \quad\left(f_{c} a_{i}+T_{i}^{1}\right) \delta_{1 i}^{-}=0, \\
\left(f_{t} a_{i}-T_{i}^{2}\right) \delta_{2 i}^{+}=0, \quad\left(f_{c} a_{i}+T_{i}^{2}\right) \delta_{2 i}^{-}=0, \\
a_{i}\left[e f l_{i}-f_{l}\left(\delta_{1 i}^{+}+\delta_{2 i}^{+}\right)-f_{c}\left(\delta_{1 i}^{-}+\delta_{2 i}^{-}\right)\right]=0 .
\end{gathered}
$$

Equation (8) shows that both problem will have the same value for the optimum; (9) shows that either $\delta_{1 i}^{+}\left(\delta_{2 i}^{+}\right)$or $\delta_{1 i}^{-}\left(\delta_{2 i}^{-}\right)$will be zero at the optimum; finally (10) indicates that the members of the structure (with nonzero area) are selected from those for which (7) is satisfied as an equation.

The problem (5)-(7) can now be interpreted in the following manner. $u_{i}^{1}, u_{i}^{2}$ are the virtual displacements at all possible joints of the structure imposed for the loading systems; the corresponding changes in length of a member $l_{i}$ is given by $\delta_{1 i}=\delta_{1 i}^{+}-\delta_{1 i}^{-}$ and $\delta_{2 j}=\delta_{2 j}^{+}-\delta_{2 i}^{-}$. The strains corresponding to these deformations will be $\epsilon_{i}^{1}=\delta_{1 i} / l_{i}$ and $\epsilon_{j}^{2}=\delta_{2 i} / l_{i}$. Equation (7) implies the following restriction on the strain systems of the deformation fields:

where

$$
f_{\iota}\left(\epsilon_{+}^{1}+\epsilon_{+}^{2}\right)+f_{c}\left(\epsilon_{-}^{1}+\epsilon_{-}^{2}\right) \leq e f,
$$

$$
\begin{aligned}
\epsilon_{+}^{i} & = \begin{cases}\epsilon^{i} & \text { if } \quad \epsilon^{i} \geq 0 \\
0 & \epsilon^{i}<0\end{cases} \\
\epsilon_{-}^{i} & =\left\{\begin{array}{ll}
-\epsilon^{i} & \epsilon^{i} \leq 0 \\
0 & \epsilon^{i}>0,
\end{array} \quad i=1,2 .\right.
\end{aligned}
$$

Furthermore, (9) shows that the strains $\epsilon_{i}^{1}, \epsilon_{i}^{2}$ will agree in sign with those of the corresponding $T_{i}^{1}, T_{i}^{2}$.

If a member of the optimum design is fully stressed under both loading systems, the restriction on the strains is governed by (11). But if a member of the optimum design is fully stressed for the loading $F_{i}^{1}$ only, then (9) and (11) imply

$$
f_{t} \epsilon_{+}^{1}+f_{c} \epsilon_{-}^{1}=e f, \quad \epsilon^{2}=0 .
$$


Similarly, if a member of the optimum design is fully stressed under the loading $F_{i}^{2}$ only, then

$$
f_{\iota} \epsilon_{+}^{2}+f_{c} \epsilon_{-}^{2}=e f, \quad \epsilon^{1}=0 .
$$

The condition (11) can be viewed as a special case of (1). The novelty of this approach is that it shows (11) is not only a sufficient condition but also a necessary condition for optimum. ${ }^{1}$

2. As an example, an optimum design in plane stresses is now given, which is the true optimum for both framework design and disc design. Tresca's yield criteria with yield stress $f$ in both tension and compression will be used.

Consider an annular disc with rigid supports along its inner circumference $r=a$ and loaded by either uniform pressure of $P$ per unit length or uniform tangential traction $T$ per unit length at the outer edge $r=b$, Fig. a.

For the uniform pressure $P$ (loading 1 ) alone, the optimum framework design consists of radial bars in compression, which is in fact a disc design with thickness distribution (see [6])

$$
h_{1}=P b / f r .
$$

For the uniform tangential traction $T$ (loading 2) alone, the optimum framework design consists of tension and compression members lie along lines of equi-angular spirals ([7], see also Fig. a) which formed a disc with thickness distribution

$$
h_{2}=2 T b^{2} / f r^{2} \text {. }
$$

If $P \leq 2 T$, the design $h_{2}$ will be capable of carrying either loading and is therefore the optimum of the present problem. Also, if $P \geq 2 T b / a$, the design $h_{1}$ will be capable of carrying either loading and is the optimum. Assume

$$
2 T b / a>P>2 T,
$$

so that both loadings have influence on the optimum design. It seems natural to examine whether the design

$$
h=\max \left(h_{1}, h_{2}\right)
$$

will give the optimum solution, Fig. b.

With reference to polar coordinates $(r, \theta)$, the radial and tangential virtual displacements will be denoted by $u_{\mathrm{r}}$ and $u_{0}$. The strains are then given by

$$
\epsilon_{r}=\frac{\partial u_{r}}{\partial r}, \quad \epsilon_{\theta}=\frac{1}{r} \frac{\partial u_{\theta}}{\partial \theta}+\frac{u_{r}}{r}, \quad 2 \gamma=\frac{1}{r} \frac{\partial u_{r}}{\partial \theta}+\frac{\partial u_{\theta}}{\partial r}-\frac{u_{\theta}}{r} .
$$

For loading 1, the portion of the disc $c<r \leq b$ is assumed to be at the point of collapse at the stress point $\sigma_{r}^{1}=-f, \sigma_{\theta}^{1}=0$, and the portion of the disc $a \leq r<c$ is below yielding, where $c=2 T b / P$ (see Fig. b). In accordance with (13), the virtual displacements are taken to be

$$
\begin{aligned}
& u_{\theta}^{1}=0, \quad u_{r}^{1}=-e(r-c), \quad r \geq c, \\
& =0, \quad r \leq c \text {. }
\end{aligned}
$$

${ }^{1}$ The author is indebted to Professor W. S. Hemp for this remark. 


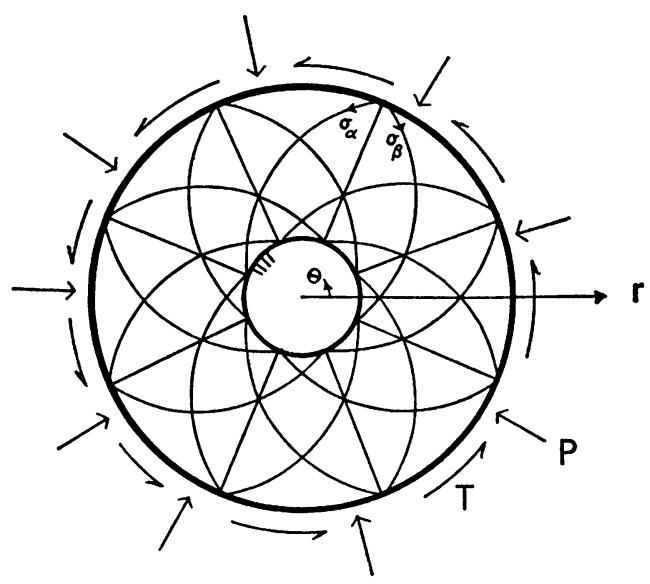

Fig. a

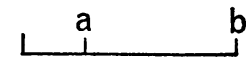

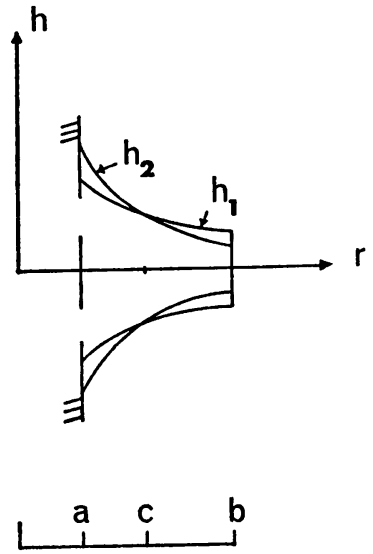

Fig. b

For loading 2, the portion of the disc $c<r \leq b$ is below yielding and the portion of the disc $a \leq r<c$ is at the point of collapse at the stress point $\sigma_{\beta}-\sigma_{\alpha}=f$, where the principle stresses $\sigma_{\alpha}, \sigma_{\beta}$ lie along lines of equi-angular spirals. In accordance with (12) the virtual displacements are taken to be

$$
\begin{aligned}
& u_{r}^{2}=0, \quad u_{\theta}^{2}=2 e r \ln (c / a), \quad r \geq c, \\
& =2 e r \ln (r / a), \quad r \leq c .
\end{aligned}
$$

Eq. (1) is found to be satisfied as follows:

$$
\begin{array}{rlrl}
D\left(\epsilon_{i j}^{1}\right)+D\left(\epsilon_{i j}^{2}\right) & =\quad \sigma_{r}^{1} \epsilon_{r}^{1}=e f, & & \geq c, \\
& =\left(\sigma_{\beta}-\sigma_{\alpha}\right) \gamma=e f, \quad r \leq c .
\end{array}
$$

The design $h$ is then proved to be the optimum since it associates with deformation modes which satisfy (1). The volume of the design $h$ can be obtained from (8), which gives

$$
V=2 \pi b[P(b-c)+2 T b \ln (c / a)] / f .
$$

\section{BIBLIOGRAPHY}

[1] L. C. Schmidt, J. Mech. Phys. Solids 10, 139-149 (1962)

[2] R. T. Shield, ZAMP 14, 38-45 (1963)

[3] R. T. Shield, Proc. 2nd Symp. Naval Struct. Mech., 1960, pp. 584-590

[4] W. S. Dorn, R. E. Gomory, H. J. Greenberg, J. Mécanique 3, 25-52 (1964)

[5] G. Zoutendijk, Methods of feasible directions, Elsevier, Amsterdam, 1960, p. 19

[6] D. C. Drucker, R. T. Shield, Proc. 9th Inter. Congr. Appl. Mech., Band 5, Brussels, 1957, pp. 212-222

[7] W. S. Hemp, College of Aeronautics Report 115, Cranfield, 1958 